\title{
A new approach to enhance the accuracy of selective harmonics-elimination technique for digital controllers
}

\author{
Hany A. Hamed ${ }^{*}$, Fatima N. Al Mansoori ${ }^{*}$ Ehab Bayoumi $^{* *} *$
}

\begin{abstract}
The selective harmonic elimination (SHE) is a preferable modulation technique in medium and high power converters applications as it enables for using low switching frequency with high flexibility to eliminate specific harmonics. The digital implementation of the SHE is a challenging issue as it requires a very accurate generation of the switching pulses. The theoretical calculation of the switching angles cannot guarantee the same results when digitally implemented by digital controllers. The digitizing process with a relatively low sampling frequency due to the digital implementation introduces error for each applied switching angle. Consequently, the applied switching angles will not match the theoretical ones resulted in residual errors for the selected harmonics that should be eliminated. This paper presents a new approach to improve SHE accuracy by online prediction and implementing of the closest true angles to minimize the residual harmonics.

K e y w or d s: selective harmonic elimination, digital controllers, three-level neutral-point clamped converters
\end{abstract}

\section{Nomenclature}

$d_{1} \quad$ : angle distance between the theoretical switching angle and previous sampled angle

$d_{2}$ : angle distance between the theoretical switching angle and next sampled angle

$F \quad$ : the objective function of the Genetic Algorithm

$k$ : number of switching angles in the quarter fundamental cycle

$f_{0} \quad$ : fundamental frequency

$f_{s} \quad$ : sampling frequency

$V_{h}$ : harmonic order $h$ amplitude

$a_{i} \quad$ : SHE angles

$a_{i}^{*} \quad$ : interpolated switching angles

$a_{i}^{\prime} \quad$ : implemented angles when digitally implemented

$a_{i}^{n-1}$ : previous sample for angle

$a_{s}^{n+1}:$ next sample for angle

$\alpha_{k}$ : implemented sampled angles when SHE is digitally implemented

$\Delta \alpha_{s}:$ minimum digitized angle step

$\Delta \alpha_{i}$ : error in SHE angles due to digital implementation.

$\theta_{\text {syn }}$ : synchronization signal

$T_{s} \quad$ : sampling time

$V_{h}^{\prime}$ : harmonic order $\mathrm{h}$ amplitude after digital implementation SHE

$\Delta V_{h}$ : error in harmonic amplitude due to digitalization

\section{Introduction}

The intensive use of power converters in the medium voltage range has introduced several challenges to electrical grids. Among those challenges, the converter emerged low order harmonics can impact the power system significantly. The low order harmonics can be mitigated by installing tuned passive filters. However, the size reduction of such filters as well as to meet the power quality standards are challenging topics. The Selective Harmonic Elimination technique (SHE) is preferable for its efficient harmonic elimination feature as well as working with low switching frequency. Back-to-back bidirectional AC-AC converter is one of the typical applications in the medium voltage range that uses SHE modulation technique [1] as shown in Fig. 1. The grid-connected converter can be modulated using various techniques such as carrier-based PWM techniques or Space Vector Modulation [2]. SHE is one of the effective modulation techniques that can eliminate a wide range of harmonics while employing a low switching frequency [3]. This modulation method is developed early in the 1960s when it is found that some low order harmonics can be eliminated by introducing several notches to a square wave [4]. Afterward, a Fourier analysis method was used to mathematically present the harmonics content in the modulated waveform by solving a group of transcendental non-linear equations semiseriously for obtaining the switching angles [5]. The main idea is to present each unwanted harmonic in its Fourier equation as a function of the modulated wave switching angles then simultaneously solve the obtained equations to produce the optimum switching angles. SHE, in general, offers low dynamic performance, on the other hand, the lower switching frequency which is commonly less than $1.5 \mathrm{kHz}$ results in reducing converter switching losses. SHE concept can be applied to various converter output waveforms such as bipolar and unipolar [6], stepped or PWM multilevel waveforms [7-9]. SHE technique is utilized in various multilevel converter topologies such as H-bridge [8-11], Modular Multilevel Converters

*Department of Electrical and Automation Engineering, Emirates Steel, Abu Dhabi, 9022, UAE, **Electrical and Electronics Engineering Department, University of Eswatini, Kwaluseni M201, Eswatini, ehab.bayoumi@gmail.com

DOI: $10.2478 /$ jee-2019-0067, Print (till 2015) ISSN 1335-3632, On-line ISSN 1339-309X 


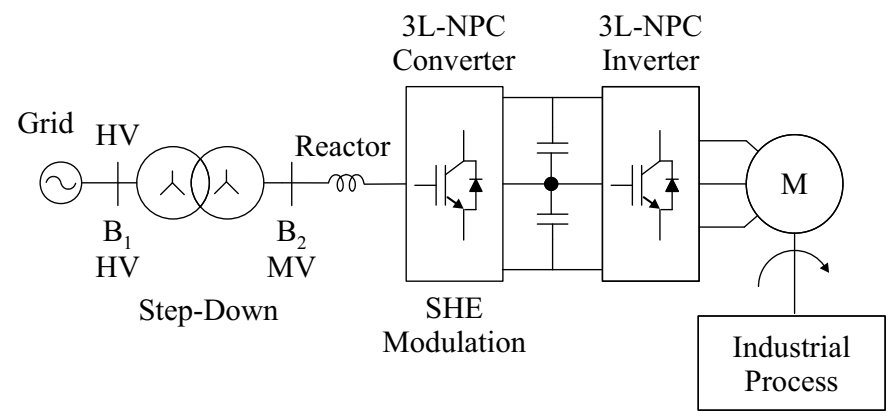

Fig. 1. Back-to-back converter configuration using SHE and PWM modulation

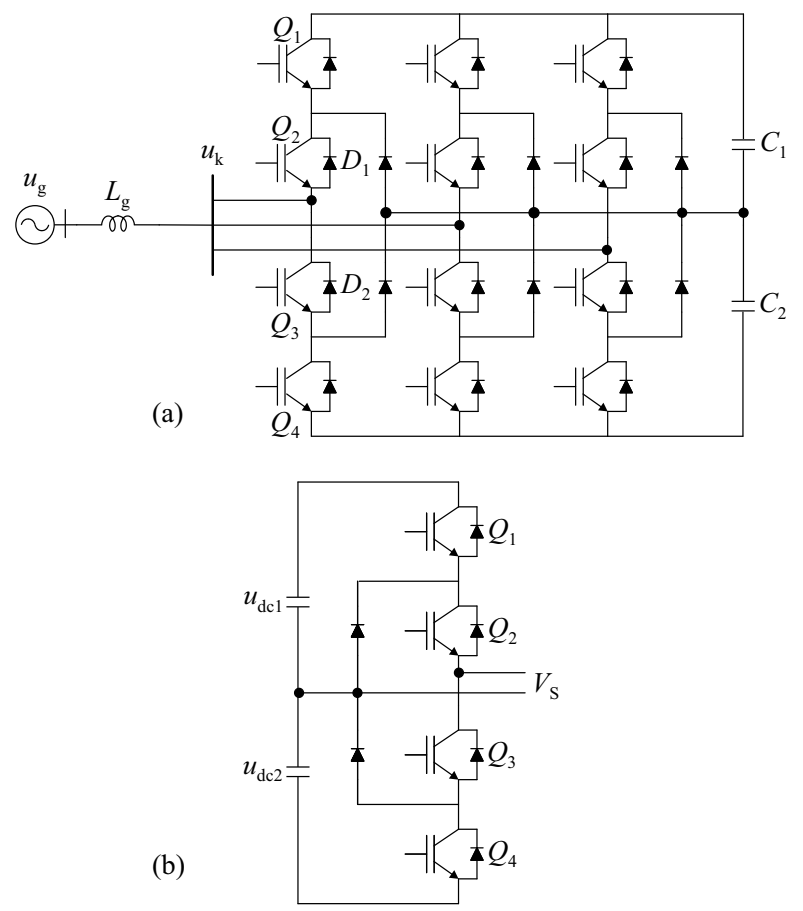

Fig. 2. 3l-NPC converter architecture.(a)-three-phase topology, (b)-single-phase topology

(MMCs) $[12,13]$, and Neutral Point Clamped Converters (NPC) $[14,16]$.

It is always challenging to find a solution of the harmonic equations for every modulation index in range from 0 to 1 . The harmonic equations can be considered as an optimization problem to find the optimum switching angles that eliminate specific harmonics. Numerous optimization techniques are typically used to obtain the switching angles, such as iterative approaches [17], Newton-Raphson method $[12,18]$, and search and optimization techniques [19-22]. The use of artificial intelligence is also presented in [23]. Other attempts to use advanced searching techniques are also introduced to reduce the computational burden [24]. Genetic algorithm (GA) is one of the efficient algorithms which is extensively used due to its flexibility in finding various solutions $[11,14,25-28,37]$.

Early in 2002, the SHE technique was proposed to modulate a 3L-NPC inverter-fed induction motor under open-loop v/f control [29]. To cover a wide range of modulation indices, the interpolation technique is proposed in [30]. The digital implementation of SHE technique especially if the speed or the capacity of the digital controller are limited due to hosting other converter control algorithms introduces a significant limitation for the maximum allowed sampling frequency. This will affect the accuracy of SHE technique as the target harmonics will not be eliminated. Moreover, the limited storage space forces to reduce the stored values of the modulation indices. Therefore, online interpolation techniques are mandatory to produce the non-tabulated modulation indices switching angles. In such case, either to use a powerful digital controller or to increase the accuracy of the SHE technique for real-time implementation [31,32]. The optimum solution of the accuracy problem is to use FPGA for the implementation of the SHE modulation algorithm while the microcontroller performs the other control functions [33]. In such case, FPGA will receive the switching angles from the control algorithm and accurately generate the switching pulses [34].

In this paper, a method to improve the accuracy of the SHE technique is proposed. This modulation technique and switching angles calculation using the genetic algorithm (GA) are presented for NPC converter topology. The proposed technique to increase the SHE method accuracy is demonstrated and simulation results are compared with the traditional SHE. The experimental result to validate the proposed technique are given.

\section{Neutral point diode clamped converters topology with SHE}

\subsection{Three-level neutral point clamped topology modula- tion}

In the last few years, there is a growing interest in multilevel topologies for medium voltage grid-connected applications. Among these topologies, Neutral Point Clamped (NPC) converters have gained much attention as they became one of the main choices for leading manufacturers of medium voltage drives. NPC's typical structure is shown in Fig. 2. The NPC converters are typically modulated by Sinusoidal Pulse Width Modulation (SPWM), Space Vector Modulation (SVM), and Selective 
Voltage

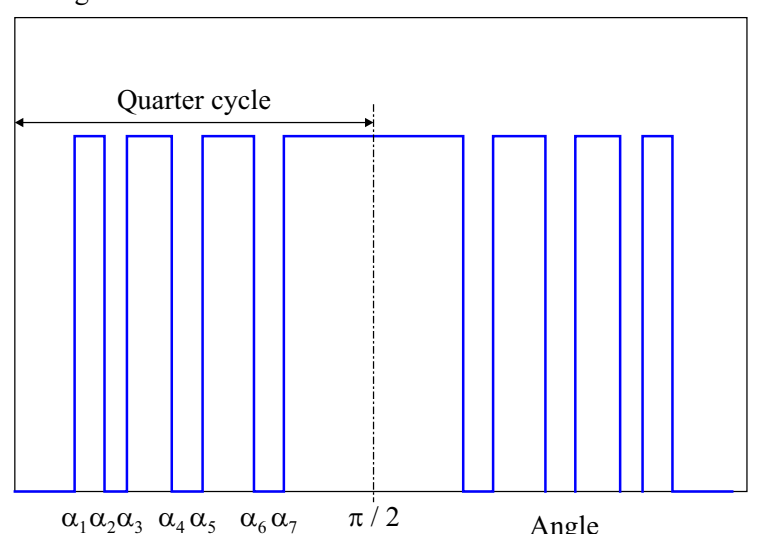

Fig. 3. The output voltage of NPC inverter with SHE method

Table 1. GA parameters for SHE optimization problem

\begin{tabular}{ll}
\hline Number of variables & 7 \\
Constraint & Function \\
Population creation & 100 \\
Creation function & constraint dependent \\
Initial range & {$[0, \pi / 2]$} \\
Fitness function & rank \\
Selection function & tournament \\
Reproduction & $0.05 \times$ Population size \\
Crossover fraction & 0.15 \\
Mutation method & adaptive feasible \\
Crossover method & two points \\
\hline Migration & forward with 0.2 \\
& fraction factor \\
\hline Non-linear & augmented Lagrangian \\
Constraint algorithm & augmented Lagrangian \\
Stopping criteria & fitness value below $10^{-6}$. \\
\hline
\end{tabular}

Harmonic Elimination (SHE) techniques [2]. It is preferable to reduce the modulator switching frequency within the medium voltage range to reduce the switching losses, however, the harmonics amplitudes will increase. Unlike all modulation techniques, SHE can offer a low switching frequency and lower harmonic contents.

\subsection{Selective harmonics elimination technique}

The selection of the converter modulator relies on the application requirements and the grid quality constraint. When the design objective is to minimize the emerged harmonics with the fundamental switching frequency, the SHE modulation technique is the primary choice. SHE method has three main advantages; first, a wide range of low harmonic orders can be eliminated resulting in canceling the need to install large passive harmonic filters. Moreover, the overall losses of cables, transformer, etc, will be reduced. Second, the most important advantage is the flexibility offered to eliminate specific harmonics to avoid network resonance. In fact, to obtain the optimum
SHE switching pattern, a definite number of nonlinear equations based on Fourier analysis of the generated voltage wave must be solved to produce switching angles for each modulation index. Due to the nature of nonlinearity between the switching angles and the modulation index, many modulation indices must be calculated and stored in a lookup table. This table consumed a considerable amount of digital controller storage [35]. The typical three level NPC converter output voltage waveform is shown in Fig. 3, in which, the switching angles are only shown for the first quarter-cycle as the rest notches are just a mirror. The produced wave could have $k$ notches with angles denoted by $\alpha_{1} \ldots \alpha_{k}$ and the maximum number of harmonics that can be eliminated equals $(k-1)$. In this paper, seven switching angles will be used to eliminate the lowest 6 harmonic orders $\left(3^{\text {rd }}, 5^{\text {th }}, 7^{\text {th }}, 9^{\text {th }}, 11^{\text {th }}, 13^{\text {th }}\right)$.

\subsection{Fourier analysis of the NPC convert waveform}

The harmonics content on the wave shown in Fig. 3 can be represented by Fourier analysis as [36]

$$
V_{h}=\sum_{h=1,2,3,5, \ldots} \frac{4 V_{d c}}{\pi h} \sum_{i=1}^{k}(-1)^{\lambda} \cos h \alpha_{i}
$$

where $V_{h}$ is the amplitude of the $h$ order harmonic, $k$ is the total number of notches per quarter cycle, $\alpha_{i}$ is the switching angles and $\lambda$ equal to 0 for rising edge notch and equals to 1 for falling edge notch. As mentioned before; $\mathrm{k}$ notches eliminate $k-1$ harmonic orders. Referring to Fig. 3, implementing 7 notches can eliminate up to 6 harmonic orders; $3^{\text {rd }} 5^{\text {th }}, 7^{\text {th }}, 9^{\text {th }}, 11^{\text {th }}$, and $13^{\text {th }}$. A set 7 harmonic equations can be formulated using (1), by setting $k=7, h \in 1,3,5,7,9,11,13$, as

$$
\begin{gathered}
V_{1}=\frac{4 V_{d} c}{\pi}\left(\cos \alpha_{1}-\cos \alpha_{2}+\cos \alpha_{3}-\cos \alpha_{4}\right. \\
\left.+\cos 5-\cos \alpha_{6}+\cos \alpha_{7}\right) \\
V_{3}=\frac{4 V_{d c}}{3 \pi}\left(\cos 3 \alpha_{1}-\cos 3 \alpha_{2}+\cos 3 \alpha_{3}-\cos 3 \alpha_{4}\right. \\
\left.+\cos 3 \alpha_{5}-\cos 3 \alpha_{6}+\cos 3 \alpha_{7}\right) \\
V_{13}=\frac{4 V_{d c}}{13 \pi}\left(\cos 13 \alpha_{1}-\cos 13 \alpha_{2}+\cos 13 \alpha_{3}-\cos 13 \alpha_{4}\right. \\
\left.+\cos 13 \alpha_{5}-\cos 13 \alpha_{6}+\cos 13 \alpha_{7}\right)
\end{gathered}
$$

Solving (2) for the optimum switching angles by setting $V_{1}$ equals to the target modulation index and setting $V_{3}: V_{1} 3$ equal to zero. The constraints that each angle should be between 0 and $\pi / 2,\left[\alpha_{1}<\alpha_{2}<\alpha_{3}<\alpha_{4}<\right.$ $\left.\alpha_{5}<\alpha_{6}<\alpha_{7}\right]$. In this paper, the Genetic Algorithm (GA) will be used [14]. The GA parameters are selected to minimize the iteration number for example by selecting 100 population with an initial range typically between 0 and $\pi / 2$. Regarding mutation, it is a random change mechanism for genes and due to the well-known solutions range, and the sequence of switching angles, the adaptive feasible method is used which performs the mutation with 
Table 2. Calculated switching angles by GA

\begin{tabular}{lccccccc}
\hline$M$ & $\alpha_{1}$ & $\alpha_{2}$ & $\alpha_{3}$ & $\alpha_{4}$ & $\alpha_{5}$ & $\alpha_{6}$ & $\alpha_{7}$ \\
0.1 & 21.76 & 22.65 & 44.12 & 45.71 & 66.41 & 68.49 & 88.87 \\
0.2 & 21.59 & 23.30 & 43.34 & 46.52 & 65.36 & 69.53 & 87.74 \\
0.3 & 21.10 & 23.66 & 42.45 & 47.20 & 64.24 & 70.51 & 86.60 \\
0.4 & 20.60 & 23.97 & 41.53 & 47.84 & 63.08 & 71.46 & 85.43 \\
0.5 & 20.07 & 24.22 & 40.55 & 48.40 & 61.85 & 72.37 & 84.24 \\
0.6 & 19.52 & 24.42 & 39.52 & 48.87 & 60.53 & 73.25 & 82.98 \\
0.7 & 18.94 & 24.52 & 38.43 & 49.18 & 59.09 & 74.02 & 81.63 \\
0.8 & 18.33 & 24.51 & 37.23 & 49.25 & 57.43 & 74.62 & 80.07 \\
0.9 & 17.65 & 24.29 & 35.84 & 48.84 & 55.34 & 74.63 & 77.92 \\
1 & 16.95 & 23.80 & 34.39 & 47.74 & 52.96 & 72.98 & 74.68 \\
\hline
\end{tabular}

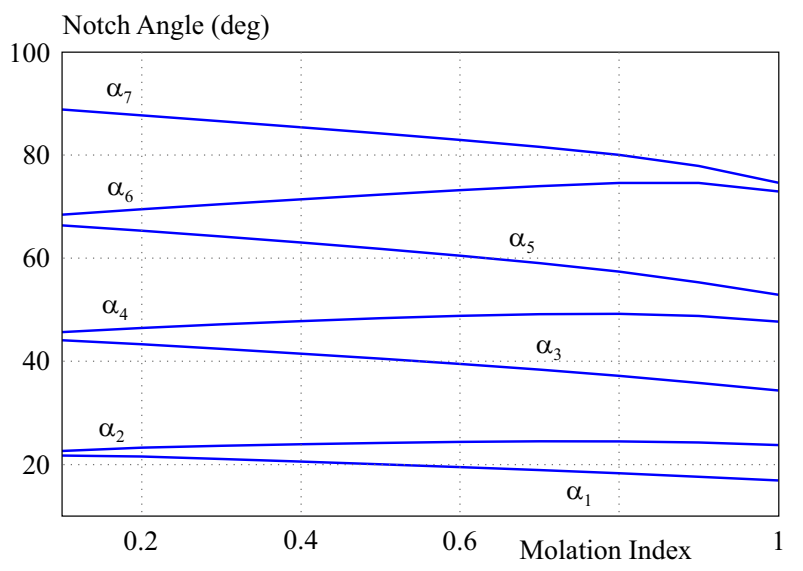

Fig. 4. Switching angles as a function of modulation index

respect to the last successful or unsuccessful generation. The GA parameters can be chosen arbitrarily depending on the optimized problem [15]. A full list of the GA parameters is illustrated in Tab. 1.

To solve (2) for each modulation index $\mathrm{m}$ in the range from 0 to 1 , the fundamental voltage $V_{1}$ is set to the target modulation index while all harmonics voltage amplitude are set to zero as

$$
\begin{aligned}
& \cos \alpha_{1}-\cos \alpha_{2}+\cos \alpha_{3}-\cos \alpha_{4}+\cos \alpha_{5} \\
& \quad-\cos \alpha_{6}+\cos \alpha_{7}=\frac{\pi}{4} m \\
& \cos 3 \alpha_{1}-\cos 3 \alpha_{2}+\cos 3 \alpha_{3}-\cos 3 \alpha_{4}+\cos 3 \alpha_{5} \\
& \quad-\cos 3 \alpha_{6}+\cos 3 \alpha_{7}=0 \\
& \cos 13 \alpha_{1}-\cos 13 \alpha_{2}+\cos 13 \alpha_{3}-\cos 13 \alpha_{4} \\
& +\cos 13 \alpha_{5}-\cos 13 \alpha_{6}+\cos 13 \alpha_{7}=0
\end{aligned}
$$

Equation (3) presents a typical optimization problem for GA. The fitness function $F$ is

$$
F=\left(\frac{m-V_{1}}{V_{1}}\right)^{2}+V_{3}^{2}+V_{5}^{2}+V_{7}^{2}+V_{9}^{2}+V_{11}^{2}+V_{13}^{2}
$$

where $V_{1}, V_{3}, V_{5}, V_{7}, V_{9}, V_{11}$, and $V_{13}$ are the amplitudes of the fundamental, $3^{\text {rd }}, 5^{\text {th }}, 7^{\text {th }}, 9^{\text {th }}, 11^{\text {th }}$, and $13^{\text {th }}$ harmonics.
The constraint function is formulated as

$$
\begin{aligned}
& 0<\alpha_{i}<\pi / 2 \\
& \alpha_{i}<\alpha_{i+1}
\end{aligned}
$$

The switching angles solution for each modulation index from 0.1 to 1 with step 0.1 is illustrated in Tab. 2 and the solution graphical presentation is presented in Fig. 4.

\subsection{Inaccuracy due to digital implementation (digitiza- tion)}

The digital implementation of the SHE modulation introduces a critical issue related to the inaccurate time implementation of the switching angles especially when the digital controller has a limitation for using high sampling frequency. This inaccuracy is due to having a difference between the calculated angles and the implemented angles. Although the calculated switching angles can be accurate enough to eliminate the selected harmonics, the error in the implemented angles introduces a relatively residual harmonics. As the digital controller sampling frequency plays a crucial role, the implemented angles must be considered when designing the SHE algorithm.

The digital implementation of SHE depends on comparing the target switching angels with a repeated digitized reference angle form $\theta_{\text {syn }}$ with a period of 0 to $2 \pi$ in order to generate the switching pulses. Due to the digitizing process, the implemented angles $\alpha_{k}$ will deviate from the calculated angles $\alpha_{i}$ by $\Delta \alpha_{k}$ which theoretically can vary from 0 to the minimum sampling angles $\Delta \alpha_{s}$.

The calculation of the minimum sampling angle angles is expressed as

$$
\Delta \alpha_{s}=360 \frac{f_{\mathrm{o}}}{f_{s}} \operatorname{deg}
$$

where $\Delta \alpha_{s}$ is the minimum sampling angle, $f_{s}$ is the sampling frequency if the digital controller, and $f_{\mathrm{o}}$ is the output voltage frequency.

The implemented angle $\alpha_{i}^{\prime}$ will always lagging the calculated angle with a value theoretically varies from 0 to 
Table 3. Modified switching angles (digitized)

\begin{tabular}{ccccccccccccccc}
\hline$m$ & $\alpha_{1}$ & $\alpha_{1}^{\prime}$ & $\alpha_{2}$ & $\alpha_{2}^{\prime}$ & $\alpha_{3}$ & $\alpha_{3}^{\prime}$ & $\alpha_{4}$ & $\alpha_{4}^{\prime}$ & $\alpha_{5}$ & $\alpha_{5}^{\prime}$ & $\alpha_{6}$ & $\alpha_{6}^{\prime}$ & $\alpha_{7}$ & $\alpha_{7}^{\prime}$ \\
\hline 0.1 & 21.76 & 22.50 & 22.65 & 23.40 & 44.12 & 45.00 & 45.71 & 45.90 & 66.41 & 66.60 & 68.49 & 69.30 & 88.87 & 89.10 \\
0.2 & 21.59 & 21.60 & 23.30 & 23.40 & 43.34 & 44.10 & 46.52 & 46.80 & 65.36 & 65.70 & 69.53 & 70.20 & 87.74 & 88.20 \\
0.3 & 21.10 & 21.60 & 23.66 & 24.30 & 42.45 & 43.20 & 47.20 & 47.70 & 64.24 & 64.80 & 70.51 & 71.10 & 86.60 & 87.30 \\
0.4 & 20.60 & 20.70 & 23.97 & 24.30 & 41.53 & 42.30 & 47.84 & 48.60 & 63.08 & 63.90 & 71.46 & 72.00 & 85.43 & 85.50 \\
0.5 & 20.07 & 20.70 & 24.22 & 24.30 & 40.55 & 41.40 & 48.40 & 48.60 & 61.85 & 62.10 & 72.37 & 72.90 & 84.24 & 84.60 \\
0.6 & 19.52 & 19.80 & 24.42 & 25.20 & 39.52 & 39.60 & 48.87 & 49.50 & 60.53 & 61.20 & 73.25 & 73.80 & 82.98 & 83.70 \\
0.7 & 18.94 & 19.80 & 24.52 & 25.20 & 38.43 & 38.70 & 49.18 & 49.50 & 59.09 & 59.40 & 74.02 & 74.70 & 81.63 & 81.90 \\
0.8 & 18.33 & 18.90 & 24.51 & 25.20 & 37.23 & 37.80 & 49.25 & 49.50 & 57.43 & 57.60 & 74.62 & 74.70 & 80.07 & 80.10 \\
0.9 & 17.65 & 18.00 & 24.29 & 24.30 & 35.84 & 36.00 & 48.84 & 49.50 & 55.34 & 55.80 & 74.63 & 74.70 & 77.92 & 78.30 \\
1.0 & 16.95 & 17.10 & 23.80 & 24.30 & 34.39 & 35.10 & 47.74 & 48.60 & 52.96 & 53.10 & 72.98 & 73.80 & 74.68 & 74.70 \\
\hline
\end{tabular}

Table 4. Calculated and digitized angles using interpolation with 0.87 modulation index

\begin{tabular}{lcccccccc}
\hline & $m$ & $\alpha_{1}$ & $\alpha_{2}$ & $\alpha_{3}$ & $\alpha_{4}$ & $\alpha_{5}$ & $\alpha_{6}$ & $\alpha_{7}$ \\
\hline Interpolated & 0.80 & 18.90 & 25.20 & 37.80 & 49.50 & 57.60 & 74.70 & 80.10 \\
angles & 0.90 & 18.00 & 24.30 & 36.00 & 49.50 & 55.80 & 74.70 & 78.30 \\
\hline (Calculated) & 0.87 & 17.85 & 24.36 & 36.26 & 48.97 & 55.97 & 74.63 & 78.57 \\
\hline $\begin{array}{l}\text { Implemented } \\
\text { angles }\end{array}$ & 0.87 & 18.00 & 25.20 & 36.90 & 49.50 & 56.70 & 74.70 & 79.20 \\
\hline
\end{tabular}

Table 5. Harmonics calculation for digitized angles with 0.87 modulation index (harmonics are \% of fundamental)

\begin{tabular}{lccccccc}
\hline $\begin{array}{l}\text { Harmonic } \\
\text { amplitude }\end{array}$ & $v_{1}$ & $h_{3}$ & $h_{5}$ & $h_{7}$ & $h_{9}$ & $h_{11}$ & $h_{13}$ \\
\hline Calculated & 0.87 & 0.0 & 0.0 & 0.0 & 0.0 & 0.1 & 0.2 \\
in $\%$ & 0.3 & 65 & 68 & 36 & 85 & 78 & 85 \\
\hline True & 0.85 & 1.5 & 1.1 & 0.3 & 0.1 & 1.9 & 2.3 \\
in $\%$ & 18 & & 1 & 4 & 5 & 3 & 9 \\
\hline
\end{tabular}

$\Delta_{s}$. Therefore, each calculated angle must be digitized and replaced with the next lagging angle. The implemented switching angles can be calculated as

$$
\alpha_{i}^{\prime}=\text { floor }\left(\frac{\alpha_{i}}{\Delta \alpha_{s}}\right) \Delta \alpha_{s}+\Delta \alpha_{s}
$$

where floor $(A)$ is the arithmetic function that rounds the elements of $Y$ to the nearest integers less than or equal to $A$.

As a result, the harmonic calculation equation presented (1) should be represented using the implemented angles. The harmonics amplitude error can be calculated using (7) and (1) as

$$
V_{h}^{\prime}=\frac{4 V_{d} c}{\pi n}\left[\sum_{i=1}^{k}(-1)^{\lambda} \cos \left(n \alpha_{i}\right)-\sum_{i=1}^{k}(-1)^{\lambda} \cos \left(n \alpha_{i}^{\prime}\right)\right]
$$

The following example presents angles digitizing error with a switching frequency equals $20 \mathrm{kHz}$, and output frequency equals to $50 \mathrm{~Hz}$. The minimum step angle can be calculated $\Delta \alpha_{s}=0.9 \mathrm{deg}$, with index equal to 0.8 from Tab. 2, $\alpha_{i}^{\prime}=18.9 \mathrm{deg}$, so, the angle error between the calculated angle and the digitized angle is $\Delta \alpha_{1}=$ $0.57 \mathrm{deg}$.

Applying the same concept to all angles in Tab. 2, the implemented switching angles are calculated as illustrated in Tab. 3.

\subsection{Inaccuracy due to interpolation}

The calculated angles usually stored in a lookup table with a limited number of modulation index values as presented in Tab. 2. If the control system requires driving the inverter with a smoothed variation of modulation index than, the switching angles should be obtained by using interpolation methods. Online linear interpolation method is used which can be expressed as

$$
\alpha_{i} *=\alpha_{n}+\left(m^{\prime}-m_{n}\right) \frac{\alpha_{n+1}-\alpha_{n}}{m_{n+1}-m_{n}}
$$

where $m_{n}$ and $m_{n+1}$ are the tabulated boundary modulation index values for the target modulation index $m$, and $\alpha_{n}, \alpha_{n+1}$ are the corresponding angles. For example, if the requested modulation index is 0.87 , the corresponding calculated and digitized angles are shown in Tab. 4.

The corresponding calculated harmonics using (2) are shown in Tab. 5. Digitizing introduces a considerable error in the calculated fundamental as well as the harmonics amplitude. To reduce the error and increase the accurateness of the SHE method, a high sampling frequency is required. A different approach to minimize the harmonics amplitude errors when the sampling frequency is limited due to the computational burden of the controller is introduced in next section. 

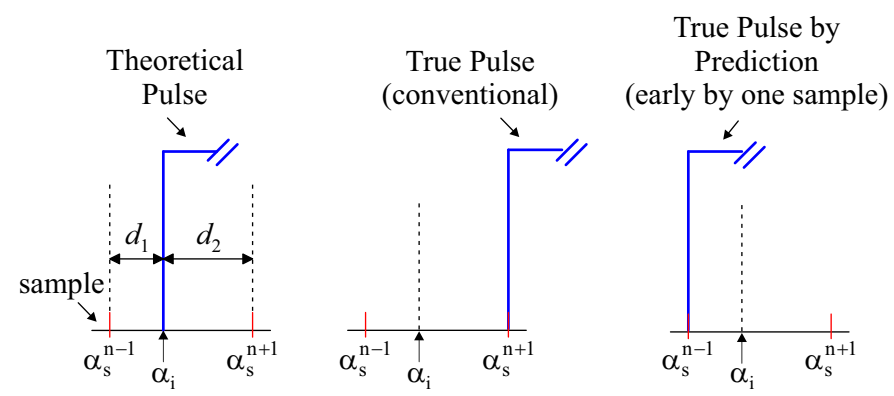

Fig. 5. Proposed PNTA method for SHE technique

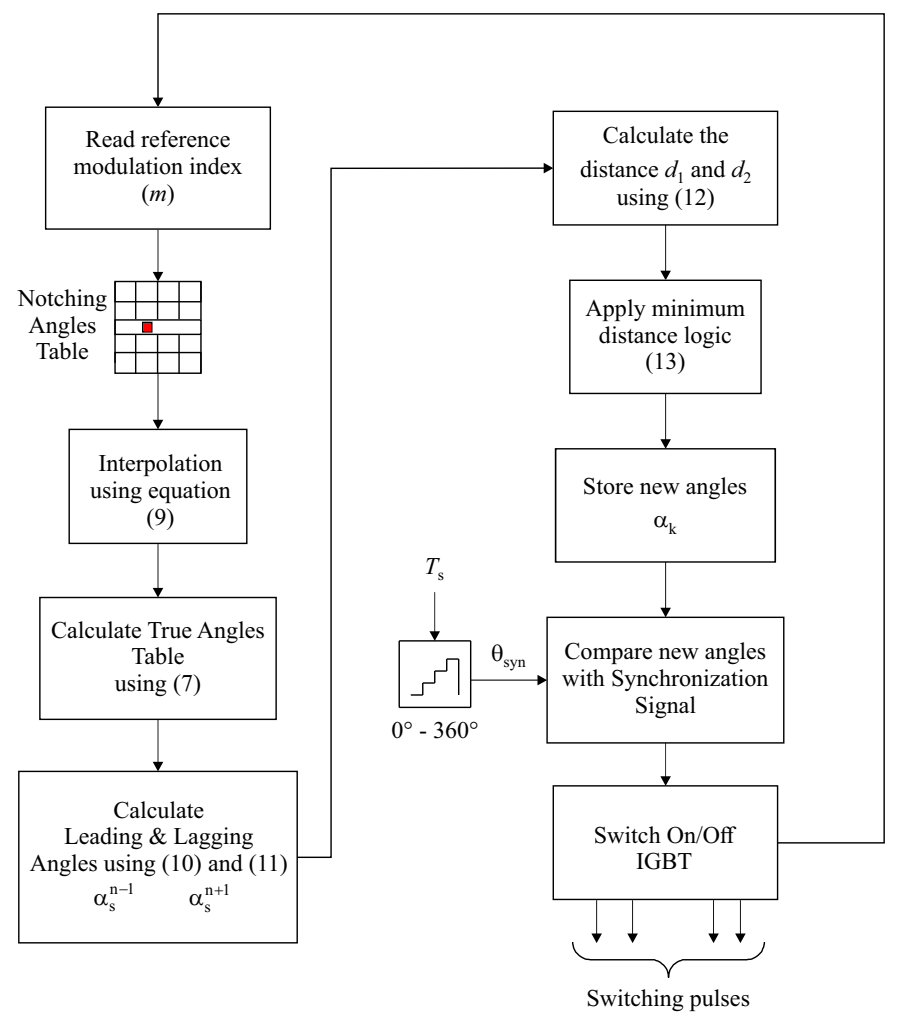

Fig. 6. Proposed PNTA algorithm flowchart

\section{Proposed predicted nearest true angle (PNTA)}

Due to the digitizing error, the implemented switching angle $\alpha_{i}^{\prime}$ could lead or lag the calculated one $\alpha_{i}$. SHE can be implemented directly using the interpolated angles illustrated in Tab. 2. However, the obtained harmonics level will have a residual error as explained before. To reduce the produced harmonic level, a new online angle correction is proposes based on predicting the nearest true angle. The Predicted Nearest Angle (PNTA) technique simply anticipates the nearest true angle to the calculated one and produce it either the angle before or the angle after $\alpha_{i}$. The algorithm principle is shown in Fig. 5. The algorithm calculates the distance between the calculated angle $\alpha_{i}$ and the two true angles which are the leading angle $\alpha_{s}^{n-1}$ and the lagging angle $\alpha_{s}^{n+1}$ then selected the nearest angle to the calculated one. The calculation of the minimum distance $d$ is as follows,

$$
\begin{gathered}
\alpha_{s}^{n-1}=\text { floor }\left(\frac{\alpha_{i}}{\Delta \alpha_{s}}\right) \Delta \alpha_{s}, \\
\alpha_{s}^{n+1}=\alpha_{s}^{n-1}+\Delta \alpha_{s}, \\
d_{1}=\alpha_{i}-\alpha_{\mathrm{s}}^{n-1}, \quad d_{2}=\alpha_{\mathrm{s}}^{n+1}-\alpha_{i} .
\end{gathered}
$$

Then select the true angle according to the following logic

$$
\left\{\begin{array}{l}
\text { if } d_{1}<d_{2} \text { then leading angle }=\alpha_{s}^{n-1}, \\
\text { if } d_{1}>d_{2} \text { then lagging angle }=\alpha_{s}^{n+1} .
\end{array}\right.
$$

The nearest true angle concept can be graphically presented as shown in Fig. 5.

The proposed algorithm flowchart is shown in Fig. 6 . The algorithm first reads the modulation index, then by using interpolation, next notch angle is calculated. The algorithm calculates the leading and the lagging true angles and selects the nearest true angle to the reference 
Table 6. Calculated and digitized angles using interpolation with 0.87 modulation index

\begin{tabular}{lccccccc}
\hline & $\alpha_{1}$ & $\alpha_{2}$ & $\alpha_{3}$ & $\alpha_{4}$ & $\alpha_{5}$ & $\alpha_{6}$ & $\alpha_{7}$ \\
\hline Interpolated (calculated): & 17.85 & 24.36 & 36.26 & 48.97 & 55.97 & 74.63 & 78.57 \\
& True & \multicolumn{7}{c}{ implemented } \\
without PNTA (digitized) & 18 & 25.20 & 36.90 & 49.50 & 56.70 & 74.70 & 79.20 \\
with proposed PNTA & 18 & 24.30 & 36.00 & 48.60 & 55.80 & 74.70 & 78.30 \\
& lagging & leading & leading & leading & leading & lagging & leading \\
\hline
\end{tabular}

Table 7. Calculated and digitized angles using interpolation with 0.87 modulation index

\begin{tabular}{lccccccc}
\hline & $v_{1}$ & $h_{3}$ & $\begin{array}{c}h_{5} \\
\%\end{array}$ & $\begin{array}{c}h_{7} \\
\%\end{array}$ & $\begin{array}{c}h_{9} \\
\%\end{array}$ & $\begin{array}{c}h_{11} \\
\%\end{array}$ & $\begin{array}{c}h_{13} \\
\%\end{array}$ \\
\hline Interpolated (calculated): & 17.85 & 24.36 & 36.26 & 48.97 & 55.97 & 74.63 & 78.57 \\
& True & & & & & & harmoics \\
\multirow{2}{*}{ without PNTA (digitized) } & 0.870 & 0.067 & 0.068 & 0.036 & 0.085 & 0.176 & 0.285 \\
with proposed PNTA & $\begin{array}{c}0.876 \\
\text { corrected }\end{array}$ & $\begin{array}{c}0.890 \\
\text { reduced }\end{array}$ & $\begin{array}{l}0.3360 \\
\text { reduced }\end{array}$ & 0.474 & 1.109 & $\begin{array}{c}0.509 \\
\text { reduced }\end{array}$ & $\begin{array}{c}1.382 \\
\text { reduced }\end{array}$ \\
\hline
\end{tabular}

Table 8. SHE pulses generation logic table

\begin{tabular}{cccc}
\hline Switching logic & Status & {$\left[Q_{1}, Q_{2}, Q_{3}, Q_{4}\right]$} & Voltage \\
\hline $0<\theta_{\text {syn }}<\alpha_{1}$ & 0 & {$[0 \mu 1 \mu 1 \mu 0]$} & 0 \\
$\alpha_{1}<\theta_{\text {syn }}<\alpha_{2}$ & 1 & {$[1 \mu 1 \mu 0 \mu 0]$} & $V_{\mathrm{dc}} / 2$ \\
$\alpha_{2}<\theta_{\text {syn }}<\alpha_{3}$ & 0 & {$[0 \mu 1 \mu 1 \mu 0]$} & 0 \\
$\alpha_{3}<\theta_{\text {syn }}<\alpha_{4}$ & 1 & {$[1 \mu 1 \mu 0 \mu 0]$} & $V_{\mathrm{dc}} / 2$ \\
$\alpha_{4}<\theta_{\text {syn }}<\alpha_{5}$ & 0 & {$[0 \mu 1 \mu 1 \mu 0]$} & 0 \\
$\alpha_{5}<\theta_{\text {syn }}<\alpha_{6}$ & 1 & {$[1 \mu 1 \mu 0 \mu 0]$} & $V_{\mathrm{dc}} / 2$ \\
$\alpha_{6}<\theta_{\text {syn }}<\alpha_{7}$ & 0 & {$[0 \mu 1 \mu 1 \mu 0]$} & 0 \\
$\alpha_{7}<\theta_{\text {syn }}<\alpha_{\pi} / 2$ & 1 & {$[1 \mu 1 \mu 0 \mu 0]$} & $V_{\mathrm{dc}} / 2$ \\
\hline
\end{tabular}

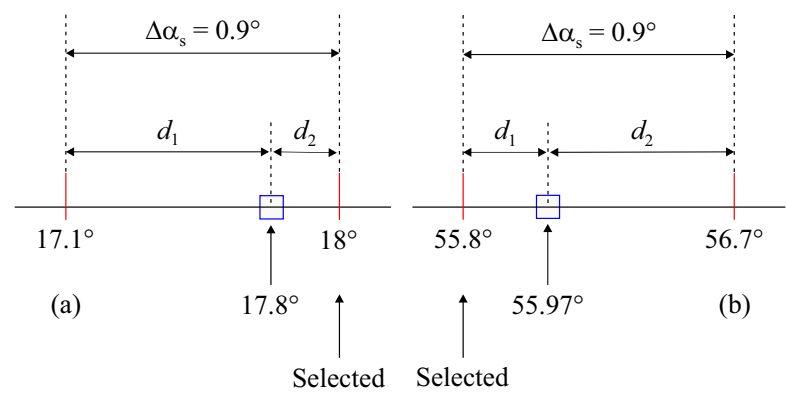

Fig. 7. Implementation of PNTA method for SHE using modulation index equals 0.87: (a) - predicted selection of $\alpha_{1}$, (b) - predicted selection of $\alpha_{s}$

one. Then, using the synchronization signal $\theta_{\text {syn }}$, the dedicated state is sent to the IGBTs to produce the required notch.

To illustrate the PNTA selection mechanism, two calculated angles are used $\alpha_{1}$ and $\alpha_{5}$ ) from Tab. 4 with modulation index equal to 0.87 . As shown in Fig. 7, the PNTA selects the lagging angle to $\alpha_{1}$ and the leading angle of $\alpha_{5}$. The PNTA results are also illustrated in Tab. 6 and Tab. 7, respectively. The harmonics generated are also presented by bar-chart as shown in Fig. 8. The switching results of implementing PNTA for all the 7 angles with 0.87 modulation index is shown in Fig. 9. Referring to Tab. 6 and Tab. 7, the proposed PNTA technique selects the lagging angle for $\alpha_{1}, \alpha_{6}$, and the leading angle for $\alpha_{2}, \alpha_{3}, \alpha_{4}, \alpha_{5}, \alpha_{7}$ as shown in Tab. 6. The PNTA effect is shown in Tab. 7 as the fundamental voltage is corrected from 0.85 to approximately 0.87 . Moreover, the odd harmonics $3^{\text {rd }}, 5^{\text {th }}, 11^{\text {th }}$ and $13^{\text {th }}$ harmonics amplitudes are reduced. The $7^{\text {th }}$ and $9^{\text {th }}$ harmonics are increased, however, they still below the permissible limits according to standards.

\section{Simulation results}

The SHE technique is implemented as shown in Fig. 10 using a single-phase Three-Level NPC inverter supplies inductive load. The notching angles illustrated in Table 2 are used. The implemented PNTA algorithm is shown previously in Fig. 6. The switching logic is illustrated in Table 8 . The total notching angles are 28 angles over each 3600 period.

The system shown in Fig. 10 is simulated with $20 \mathrm{kHz}$ sampling frequency. To investigate the accumulated error due to both digitizing and interpolation, a non-tabulated modulation index equals to 0.87 is selected referring to Tab. 4. The results of the proposed algorithm are shown in Fig. 11. The modified switching angles $\alpha_{1}, \alpha_{5}$ and $\alpha_{6}$ are shown in Fig. 11(a) compared with its original version. The improvement in individual selected harmonics is shown in Fig. 11(b). In comparison with the conventional SHE method, the proposed technique successfully reduces the selected harmonics amplitude. To validate the proposed interpolation technique, another non-tabulated modulation index equals to 0.55 is tested. The NPC is set to a $50 \mathrm{~Hz}$ reference wave using a $20 \mathrm{kHz}$ sampling frequency. The terminal voltage and current waveforms are shown in Fig. 12(a). The obtained harmonics are within the IEC standard limits which confirms the design criteria as shown in Fig. 12(b), [36]. Due to the digitizing errors 


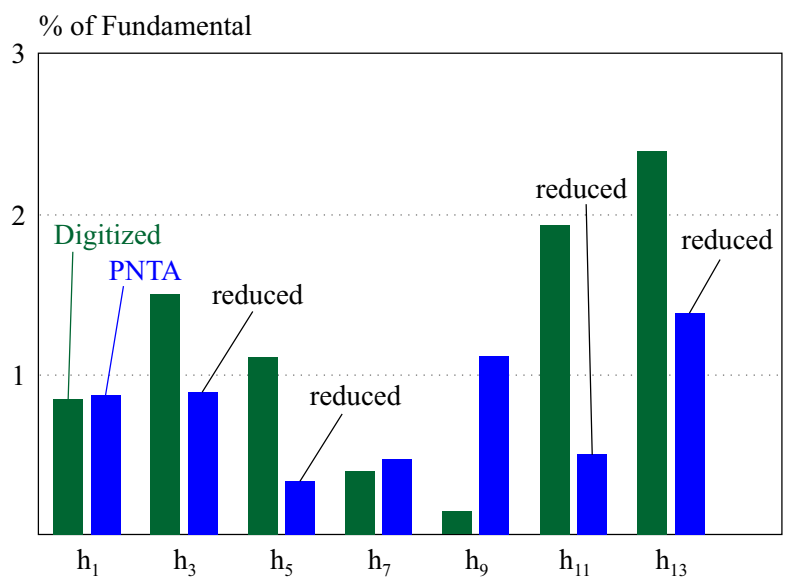

Fig. 8. Calculated harmonic using (6) for $m=0.87$

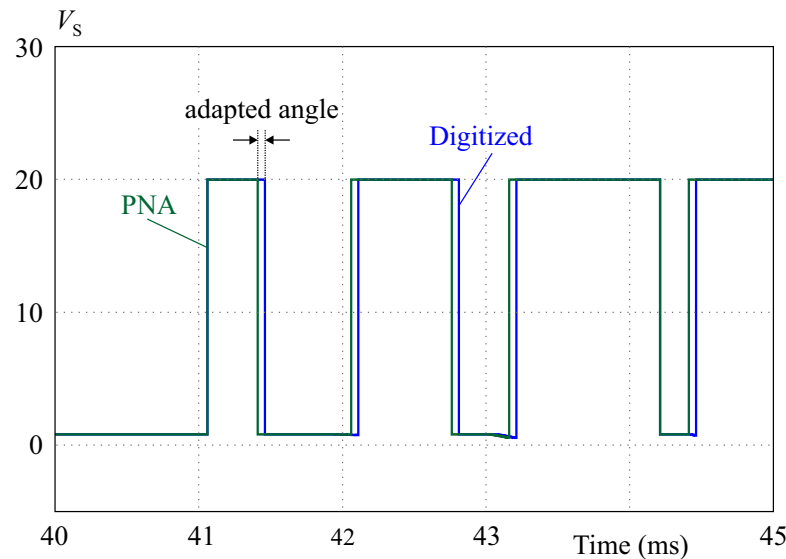

Fig. 9. The output voltage for digitized angles and PNTA technique

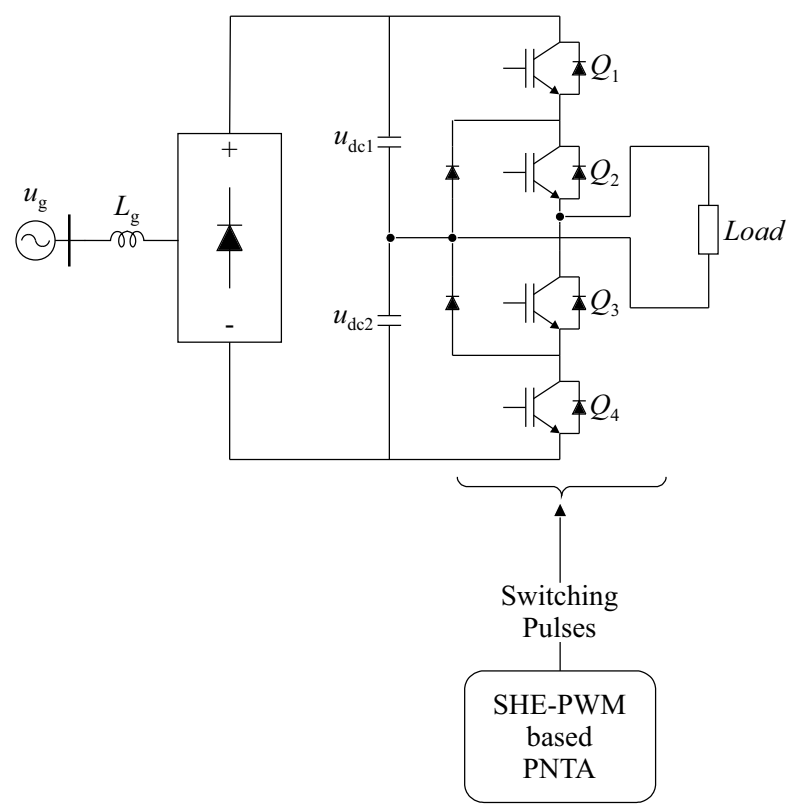

Fig. 10. Single-phase 3L-NPC inverter with PNTA based SHE

that may affect the accuracy of the SHE method, a wide range of modulation index will be tested experimentally.
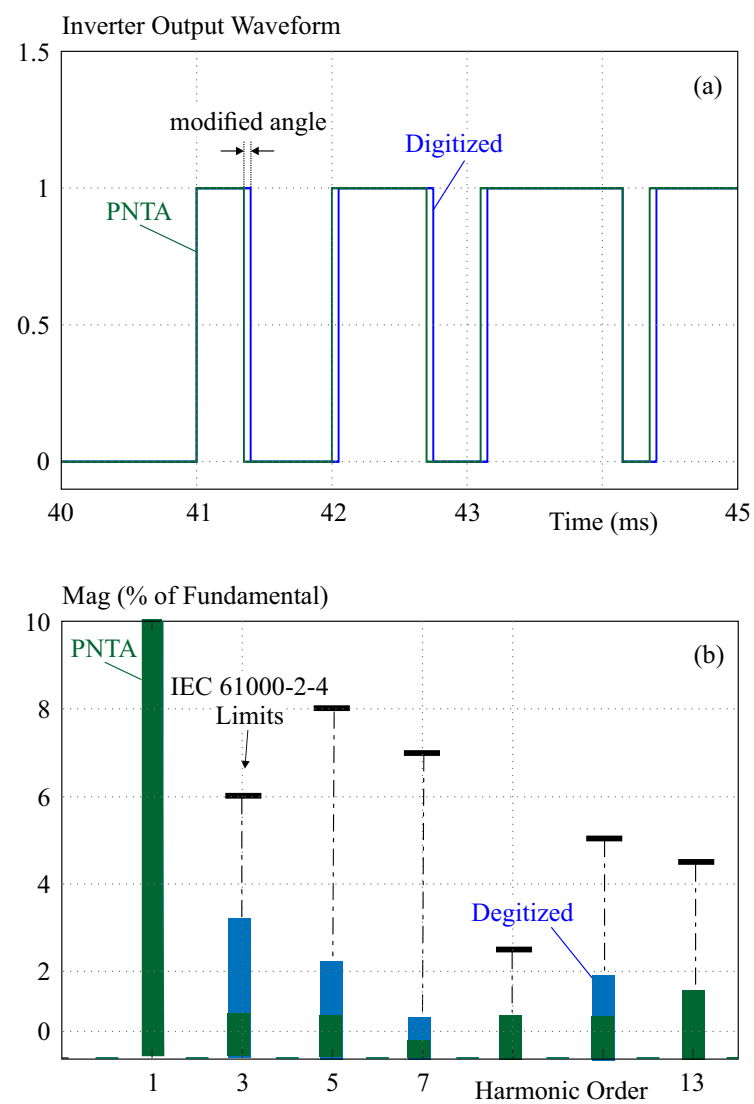

Fig. 11. Performance comparison between conventional SHE (Digitized) and PNTA technique with $m=0.87$, (a) - ideal inverter output voltage waveform, (b) - harmonic analysis comparison
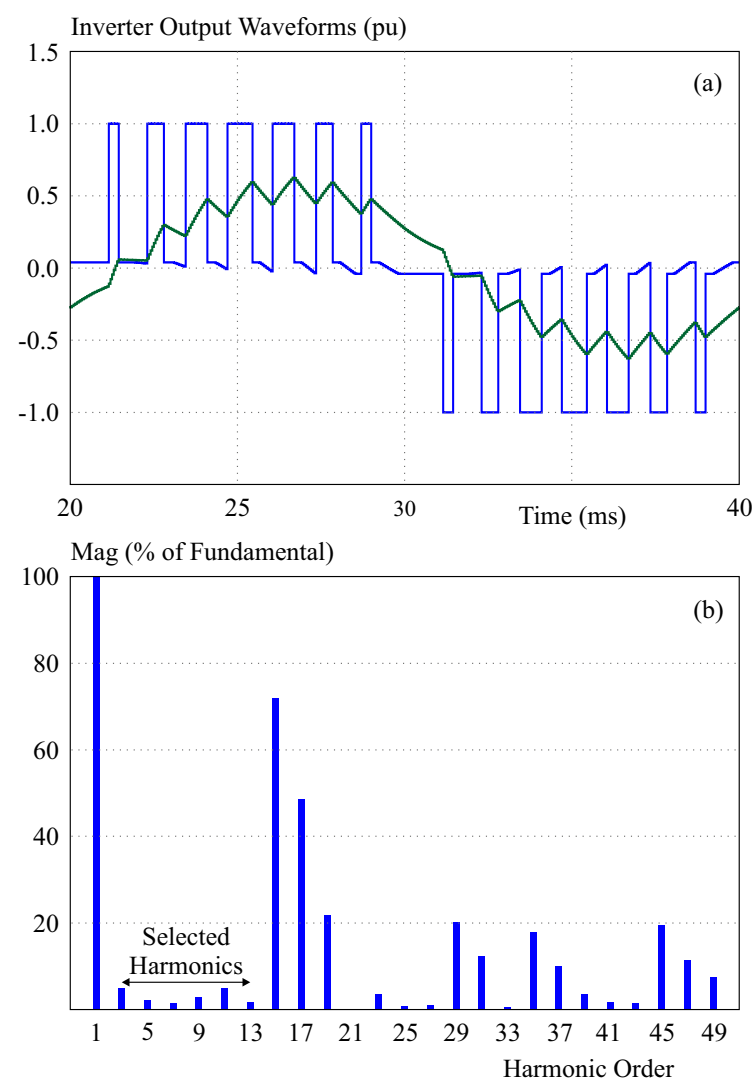

Fig. 12. Simulation results with 0.55 modulation index values: (a) voltage and current waveforms, (b) - harmonic analysis 


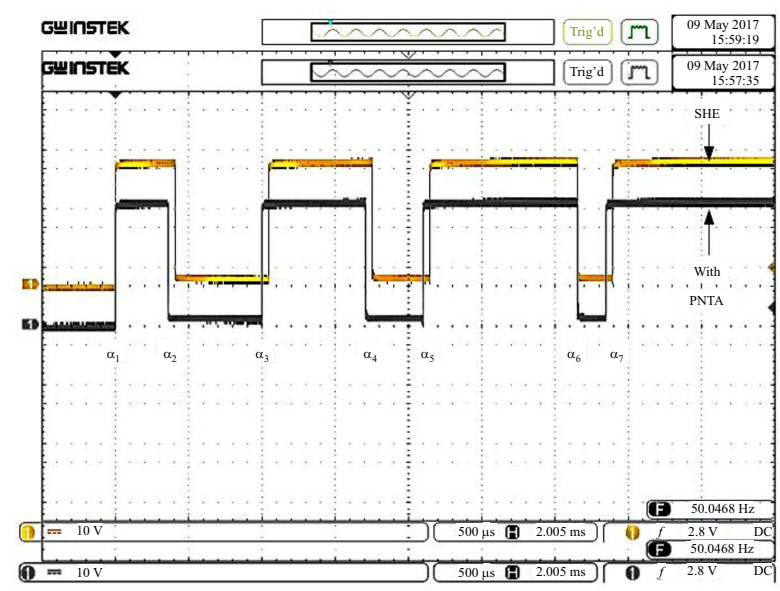

Fig. 13. Experimental results for digitized and PNTA technique

Table 9. Converter parameters

\begin{tabular}{lc}
\hline Actual DD-link voltage & $40 \mathrm{~V}$ \\
Load inductance & $1.67 \mathrm{mH}$ \\
Load resistance & $923 \Omega$ \\
DSP Sampling frequency & $20 \mathrm{kHz}$ \\
Converter total & $3.6 \mathrm{mF}$ \\
DC-link capacitors & \\
\hline
\end{tabular}

\section{Experimental results}

SHE based PNTA technique is validated by using the test setup shown in Fig. 13 which consists of single-phase $3 \mathrm{~L}-\mathrm{NPC}$ inverter supplying $R L$ load. The system parameters are illustrated in Tab. 9. The PNTA algorithm is implemented within the DSP dSPACE 1104 with a high sampling frequency $(20 \mathrm{kHz})$. The calculated switching angles shown in Tab. 2 are stored in DPS. Smoothed modulation index variation is obtained by implementing online interpolation method. The harmonics are measured by Fluke-435 power analyzer. The proposed technique is tested with a non-tabulated modulation index equals to 0.87 to include the interpolation inaccuracy. The output

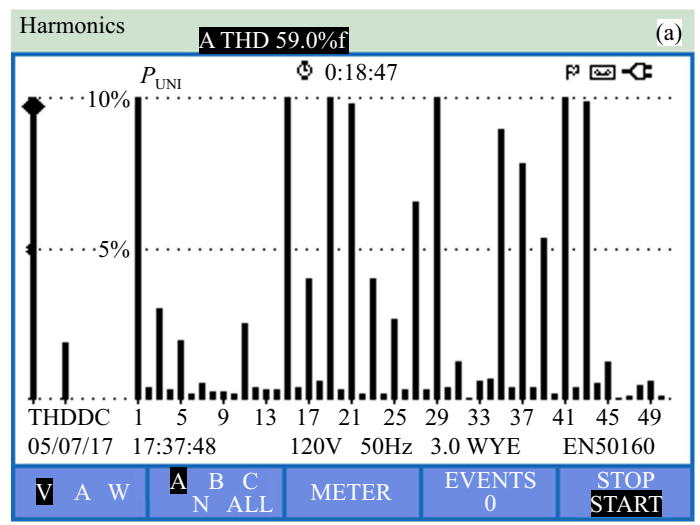

wave harmonics contents of the conventional SHE is compared with the proposed PNTA technique with a wide range of modulation indices values to validate the system accuracy.

\subsection{Performance of SHE with PNTA technique}

The results of both proposed PNTA technique and the interpolated SHE technique are compared. The PNTA technique selected the nearest next angle as shown in Fig. 13 which matched the previously simulated results shown in Fig. 11(a). In detail, the leading next angle for the notches 2, 3, 4 and 7 while the next lagging angle for notches 1 and 6 are selected. The remarkable enhancement of using the PNTA technique is shown in Fig. 14 where the low order individual harmonics are reduced compared to the conventional THD method.

\subsection{Performance of the proposed technique over a wide range of modulation indices}

The experimental test is performed with similar modulation index values used for the simulation test. Two values of non-tabulated modulation index; 0.55 and 0.87 are used. The comparison between the simulation and experimental results is shown in Fig. 15 for two modulation index values $m=0.55$ and $m=0.87$. The obtained experimental results match the simulation ones which emphasize the effectiveness of the PNTA technique to enhance the overall performance of SHE method.

To examine the accuracy of the proposed technique over a wide range of non-tabulated modulation index values, the system is tested with $0.65,0.75,0.85$ and 0.95 modulation indices. The harmonic analysis results are shown in Fig. 16. The obtained results confirm the accurateness of the interpolation technique and emphasize the effectiveness of the proposed PNTA technique.

\section{Conclusions}

The harmonics residual error due to the digital implementation of the SHE modulation technique is analyzed

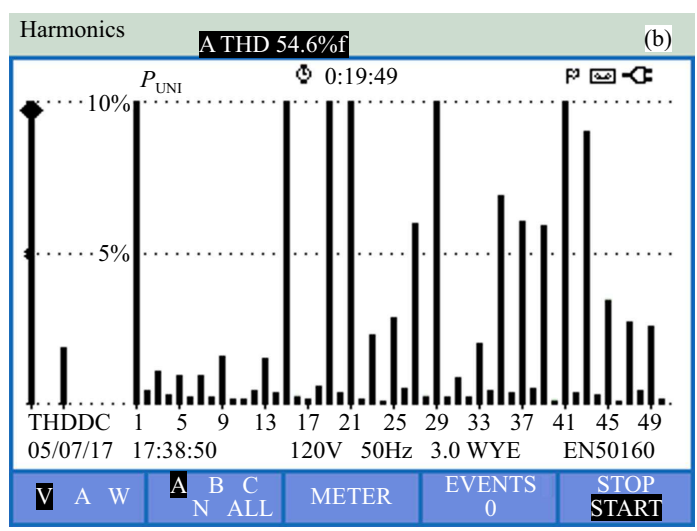

Fig. 14. Experimental results for conventional SHE and the proposed technique:(a) - conventional SHE harmonic analysis, (b) - proposed SHE harmonic 

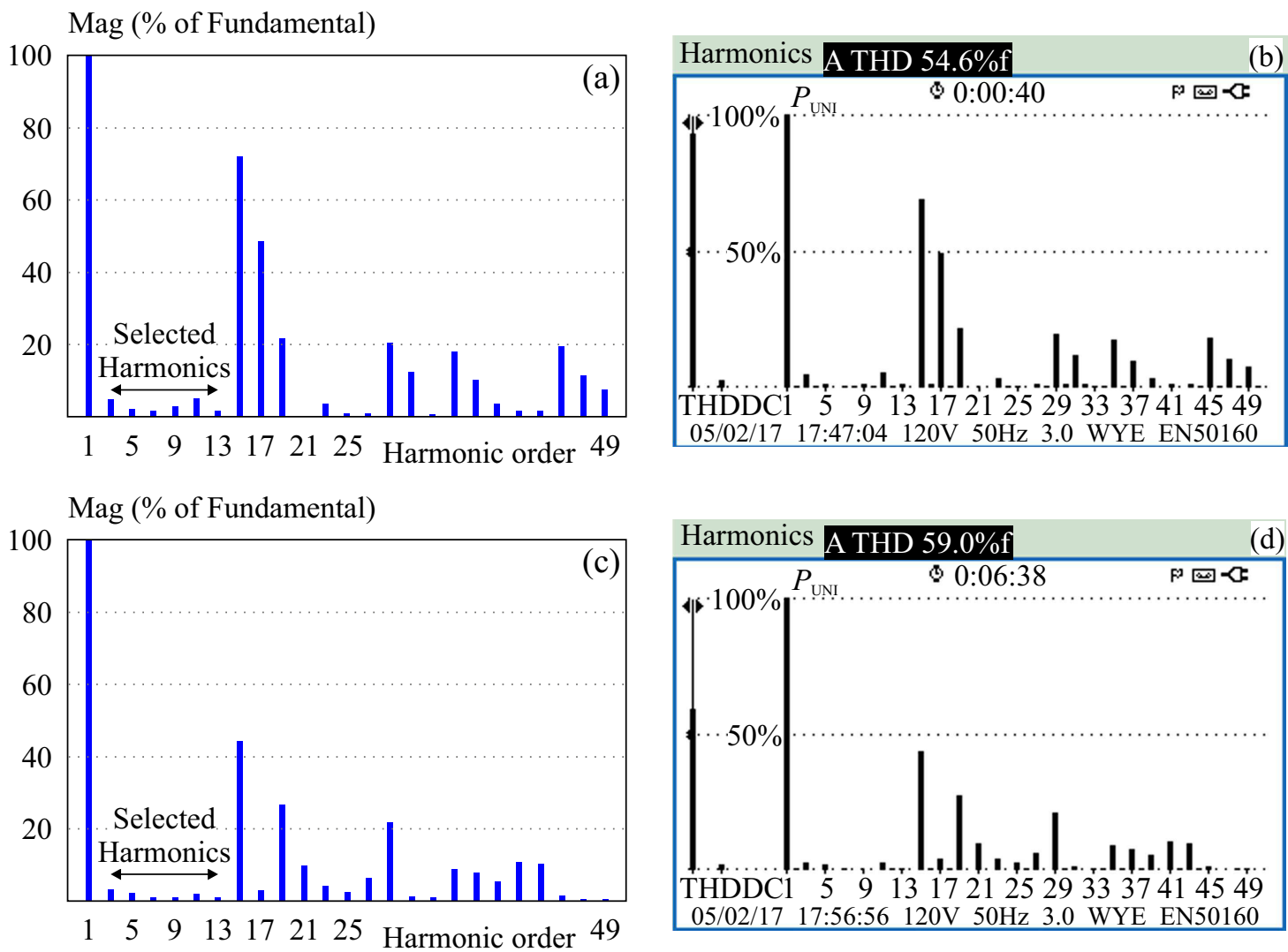

Fig. 15. Experimental results of SHE with PNTA technique for different modulation index values: (a) - simulation with $m=0.55$, (b) - experimental with $m=0.55$, (c) - simulation with $m=0.87$, (d) - experimental analysis with $m=0.87$
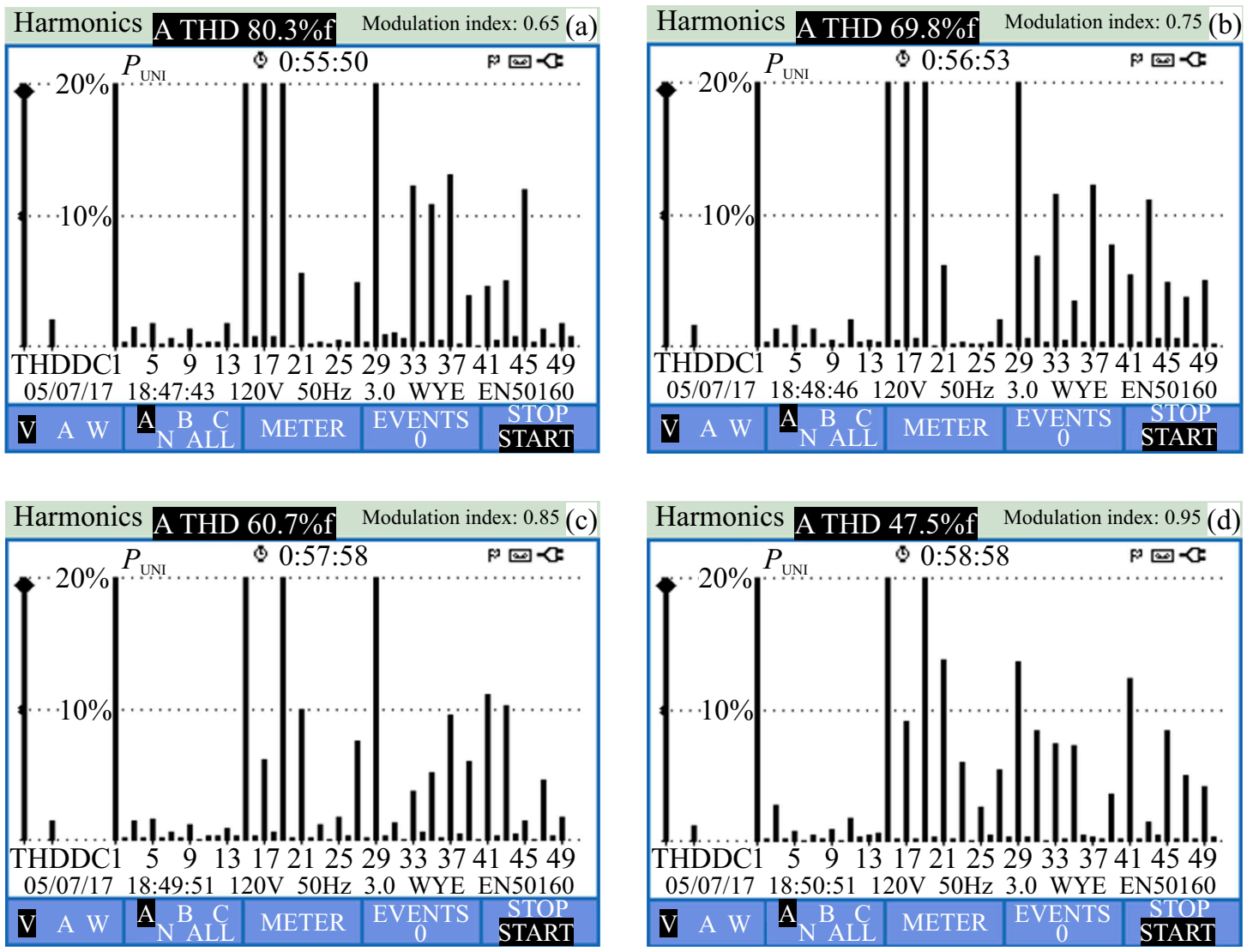

Fig. 16. Experimental results of SHE with PNTA technique with range of non-tabulated modulation index values: $($ a) $-m=0.65,(\mathrm{~b})-$ $m=0.75,(\mathrm{c})-m=0.85$, and $(\mathrm{d})-m=0.95$ 
and mitigated. The selection of the nearest sample technique is successfully implemented which resulted in reducing the error between the calculated switching angles and the true implemented ones. Consequently, the residual harmonics amplitude are reduced. The experimental test is performed with a wide range of modulation indices to validate the technique. The results showed that not all selected harmonics residual errors are reduced. Some of the residual harmonics are not reduced due to the limited controller sampling frequency. However, the overall accuracy of SHE is improved. It is concluded that to increase the accuracy of the SHE, the sampling frequency of the digital controller must be high enough or the SHE algorithm itself needs to be implemented within a separate digital controller or FPGA. Finally, based on the obtained results, the proposed SHE technique is functioning successfully with high accuracy when the digital controller sampling frequency is at the limit.

\section{REFERENCES}

[1] Y. Zhang, Z. Zhao, T. Lu, Y. Zhang, and L. Yuan, "A Novel Control Scheme for Three-Level NPC Back-to-Back Converter", 2008 IEEE Vehicle Power Propulsion Conference, pp. 1-5, September 2008.

[2] J. I. Leon, S. Kouro, L. G. Franquelo, J. Rodriguez, and B. $\mathrm{Wu}$, "The Essential Role the Continuous Evolution of Modulation Techniques for Voltage-Source Inverters in the Past, Present", IEEE Transactions on Industrial Electronics, vol. 63, pp. 2688-2701, 2016.

[3] M. S. A. Dahidah, G. Konstantinou, and V. G. Agelidis, "A Review of Multilevel Selective Harmonic Elimination PWM: Formulations, Solving Algorithms", IEEE Transactions on Power Electronics, vol. 30, pp. 4091-4106, 2015.

[4] F. G. Turnbull, "Selected Harmonic Reduction in Static dc-ac Inverters", IEEE Trans. Communication and Electronics, vol. 83, pp. 374-378, 1964.

[5] H. S. Patel and R. G. Hoft, "Generalized Techniques of Harmonic Elimination Voltage Control in Thyristor Inverters: Part I-Harmonic Elimination", IEEE Trans. on Ind, Appl., vol. IA-9, pp. 310-317, 1973.

[6] B. Wu, S. B. Dewan, and G. R. Slemon, "PWM-CSI Inverter for Induction Motor Drives", in Conference Record of the IEEE Industry Applications Society Annual Meeting 1989, pp, 508-513, vol. 1.

[7] M. S. A. Dahidah and V. G. Agelidis, "Selective Harmonic Elimination PWM Control for Cascaded Multilevel Voltage Source Converters: A Generalized Formula", IEEE Transactions on Power Electronics, vol. 23, pp. 1620-1630, 2008.

[8] S. Kundu, S. Bhowmick, and S. Banerjee, "Improvement of Power Utilisation Capability for a Three-Phase Seven-Level CHB Inverter using an Improved Selective Harmonic Elimination PWM Scheme by Sharing a Desired Proportion of Power Among the H-Bridge Cells", in IET Power Electronics, vol. 12, no. 12 , pp. $3242-3253,16.10 .2019$.

[9] M. A. Hosseinzadeh, M. Sarbanzadeh, Y. Salgueiro, M. Rivera, and P. Wheeler, "Selective Harmonic Elimination In Cascaded H-Bridge Multilevel Inverter Using Genetic Algorithm Approach", 2019 IEEE International Conference on Industrial Technology (ICIT) Melbourne, Australia, 2019, pp. 1527-1532.

[10] A. Moeini, H. Iman-Eini, and M. Bakhshizadeh, "Selective Harmonic Mitigation-Pulse-Width Modulation Technique with Variable DC-Link Voltages in Single Three-Phase Cascaded H-Bridge Inverters", IET Power Electronics, vol. 7, pp. 924-932, 2014.
[11] F. H. Dupont, J. R. Pinheiro, V. F. Montagner, and H. Pinheiro, "GA Optimization to Mitigate Voltage Harmonics of Cascade Multilevel Converters", in 2015 CHILEAN Conference on Electrical Electronics Engineering, Information Communication Technologies (CHILECON) 2015, pp. 461-466.

12] Y. Sinha and A. Nampally, "Modular Multilevel Converter Modulation using Fundamental Switching Selective Harmonic Elimination Method", 2016 IEEE International Conference on Renewable Energy Research Applications (ICRERA), pp. 736-741, 20-23 November 2016.

[13] A. Perez-Basante, S. Ceballos, G. Konstantinou, J. Pou, J. Andreu, and I. M. De Alegria, "(2N+1) Selective Harmonic Elimination-PWM for Modular Multilevel Converters: A Generalized Formulation and A Circulating Current Control Method", IEEE Transactions on Power Electronics, vol. 33, pp. 802-818, 2018.

14] A. Sharma, D. Singh, and S. Gao, "Harmonic Elimination in Three Phase Cascaded Multilevel Inverter using Genetic Algorithm", 2019 IEEE International Conference on Sustainable Energy Technologies Systems (ICSETS) Bhubaneswar, India, 2019, pp. 213-218.

[15] K. S. Neralwar, P. M. Meshram, and V. Borghate, "GA Based Hybrid Selective Harmonic Elimination (SHE) Technique Applied to Five-Level Nested Neutral Point Clamped (NNPC) Converter", 2016 IEEE 1st International Conference on Power Electronics Intelligent Control Energy Systems (ICPEICES), Delhi, 2016, pp. 1-6.

[16] S. R. Pulikanti, M. S. A. Dahidah, and V. G. Agelidis, "Voltage Balancing Control of Three-Level Active NPC Converter Using SHE-PWM", IEEE Trans. Power Del., vol. 26, pp. 258-267, 2011.

[17] T. Kato, "Sequential Homotopy-Based Computation of Multiple Solutions for Selected Harmonic Elimination in PWM Inverters", IEEE Transactions on Circuits Systems I: Fundamental Theory Applications, vol. 46, pp. 586-593, 1999.

[18] E. H. E. Aboadla, S. Khan, M. H. Habaebi, T. Gunawan, B. A. Hamidah, and M. Tohtayong, "Selective Harmonics Elimination Technique in Single Phase Unipolar H-Bridge Inverter", 2016 IEEE Student Conference on Research Development (SCOReD), pp. 1-4, 13-14 December 2016.

[19] A. Routray, R. Kumar Singh, and R. Mahanty, "Harmonic Minimization in Three-Phase Hybrid Cascaded Multilevel Inverter using Modified Particle Swarm Optimization", in IEEE Transactions on Industrial Informatics, vol. 15, no. 8, pp. 4407-4417, August 2019.

[20] P. Kumar Kar, A. Priyadarshi, and S. Bhaskar Karanki, "Selective Harmonics Elimination using Whale Optimisation $\mathrm{Al}$ gorithm for a Single-Phase-Modified Source Switched Multilevel Inverter", in IET Power Electronics, vol. 12, no. 8, pp. 1952-1963, 10.7.2019.

[21] J. Hao, G. Zhang, Y. Zheng, W. Hu, and K. Yang, "Solution for Selective Harmonic Elimination in Asymmetric Multilevel Inverter Based on Stochastic Configuration Network Levenberg-Marquardt Algorithm", 2019 IEEE Applied Power Electronics Conference Exposition (APEC) Anaheim, CA, USA, 2019, , pp. 2855-2858.

[22] M. Ahmed et al, "General Mathematical Solution for Selective Harmonic Elimination", in IEEE Journal of Emerging Selected Topics in Power Electronics, 2019.

[23] K. Yang, J. Hao, and Y. Wang, "Switching Angles Geneation for Selective Harmonic Elimination by using Artificial Neural Networks Quasi-Newton Algorithm", 2016 IEEE Energy Conversion Congress Exposition (ECCE) pp, 1-5, 18-22 Sep 2016.

[24] S. Mohammadalizadeh and M. Ghayeni, "A New Strategy in Selective Harmonic Elimination for a Photovoltaic Multilevel Inverter", 2016 Iranian Conference on Renewable Energy \& Distributed Generation (ICREDG), pp. 50-55, 2-3 April 2016. 
[25] B. Ozpineci, L. M. Tolbert, and J. N. Chiasson, "Harmonic Optimization of Multilevel Converters using Genetic Algorithms", IEEE Power Electronics Letters, vol. 3, pp. 92-95, 2005.

[26] K. L. Shi and L. Hui, "Optimized PWM Strategy Based on Genetic Algorithms", IEEE Trans, Ind, Electron, vol. 52, pp. 1458-1461, 2005.

[27] S. Ramkumar, V. Kamaraj, and S. Thamizharasan, "GA Based Optimization Critical Evaluation SHE Methods for Three-Level Inverter", in 2011 1st International Conference on Electrical Energy Systems 2011, pp. 115-121.

[28] S. Debnath and R. N. Ray, "Harmonic Elimination in Multilevel Inverter using GA PSO: A Comparison", in Electrical Electronics Computer Science (SCEECS), 2012 IEEE Students' Conference on, 2012, pp. 1-5.

[29] Y. Qingguang, S. Qiang, L. Wenhua, and L. Yongqiang, "DSP LF2407 in NPC Three-Level Inverter using Constant V/f principle SHE-PWM method", in 6th International Conference on Signal Processing 2002, pp, 1723-1726, vol. 2.

[30] J. R. Tibola, H. Pinheiro, and R. F. D. Camargo, "Closed Loop Selective Harmonic Elimination Applied to a Grid Connected PWM Converter with LCL Filter", XI Brazilian Power Electronics Conference pp, 746-752, 11-15 Sept, 2011.

[31] K. Yang et al, "Real-Time Switching Angle Computation for Selective Harmonic Control", in IEEE Transactions on Power Electronics, vol. 34, no. 8, pp. 8201-8212, Aug 2019.

[32] M. Ahmed, A. Sheir, and M. Orabi, "Real-Time Solution Implementation of Selective Harmonic Elimination of Seven-Level Multilevel Inverter", in IEEE Journal of Emerging Selected Topics in Power Electronics, vol. 5, no. 4, pp. 1700-1709, Dec 2017.

[33] V. Castiglia, R. Miceli, G. Schettino, M. G. Cimoroni, C. Buccella, and C. Cecati, "Selective Harmonic Elimination in a 5-Level Single Phase Converter with FPGA Based Controller", 2018 5th International Symposium on Environment-Friendly Energies Applications (EFEA) Rome, 2018, pp. 1-6.

[34] S. Ahmad, Z. A. Ganie, I. Ashraf, and A. Iqbal, "Harmonics Minimization in 3-Level Inverter Waveform its FPGA Realization", 2018 3rd International Innovative Applications of Computational Intelligence on Power Energy Controls with their Impact on Humanity (CIPECH), Ghaziabad, India, 2018, pp. 1-5.

[35] Y. Zhang, Y. W. Li, N. R. Zargari, and Z. Cheng, "Improved Selective Harmonics Elimination Scheme With Online Harmonic Compensation for High-Power PWM Converters", IEEE Trans, Ind, Electron, vol. 30, pp. 3508-3517, 2015.

[36] M. Mitchell "An Introduction to Genetic Algorithms", MIT Press, 1998

[37] IEC 61000-2-4-2002 "Electromagnetic compatibility", (EMC) - Part 2, Environment-Section 4, Compatibility Levels in Industrial Plants for Low-Frequency Conducted Disturbances STANDARDS, 2002.

Received 16 July 2019

Hany A. Hamed received the BSc MSc, and PhD from Menoufia University, Egypt in 1994, 2000 and 2018 respectively. Since 1997 he has been working as an Electrical and Automation Engineer for various industries. He participated in several projects including high-performance drives tuning, grid-connected converters installation and tuning, harmonics mitigation techniques, and artificial intelligence application. He is currently with Emirates Steel, UAE as head of the Electrical and Automation department. His area of interest includes multilevel inverters, HVDC and PV power electronic applications, high-performance drives, grid-connected multilevel converters, modulation strategies, and synchronization techniques.

Fatima N. Al Mansoori received Bachelor of Applied Science major on Electrical Engineering Technology from Higher Collages of Technology, Abu Dhabi, UAE in 2018. She is currently with the Electrical and Automation Department, Emirates Steel, UAE. Her research interests include electrical drives and their application in industrial field, power electronics applications, modulation strategies, power converters control, and renewable energy.

Ehab H. E. Bayoumi received his BSc, MSc and PhD degrees in electrical power engineering from Helwan University Ain Shams University and Cairo University, Egypt in 1988, 1996 and 2001 respectively. He has been with the Electronic Research Institute (ERI), Cairo, Egypt, since 1990. From 2000 to 2001 he joined LUT, Finland, as a Visiting Researcher. He was appointed as an Assistant Professor at Chalmers University of Technology, Sweden from 2003 to 2005. From 2005 to 2010 he was Appointed as Associate Professor in ERI Egypt. In 2010 he was appointed as Full Professor in ERI Egypt. Currently, he is a Full Professor in University of Eswatini, Eswatini. He also served at Yanbu Industrial College, Saudi Arabia and Higher Collages of Technology, United Arab Emirates. His research interests include high-performance ac machines, power quality, switching power converters, DSP-based control applications, and nonlinear control applications in power electronics, smart-grid, and electric drive systems. Prof. Bayoumi was appointed as an Editor-in Chief of the International Journal in Industrial Electronics and Drives in 2013. 